\title{
Investigation of Various Metamaterial Structures Using Multilevel Fast Multipole Algorithm ${ }^{\dagger}$
}

\author{
Özgür Ergül ${ }^{1}$, Çağlar Yavuz ${ }^{1}$, Alper Ünal ${ }^{1}$, and Levent Gürel*1,2 \\ ${ }^{1}$ Department of Electrical and Electronics Engineering \\ ${ }^{2}$ Computational Electromagnetics Research Center (BiLCEM) \\ Bilkent University, TR-06800, Bilkent, Ankara, Turkey \\ E-mail: \{ergul,lgurel\}@ee.bilkent.edu.tr
}

\section{Introduction}

We consider accurate simulations of metamaterial (MM) structures consisting of splitring-resonators (SRRs) and thin wires (TWs). We employ electric-field integral equation (EFIE) [1] to formulate the scattering problems involving these complicated structures. Accurate modelling of MMs translates into very large computational problems, which can be solved with the aid of advanced acceleration techniques, such as the multilevel fast multipole algorithm (MLFMA) [2]. We investigate various multilayer structures of SRRs as well as composite metamaterials (CMMs) constructed by the arrangements of SRR and TW arrays. In addition, we consider various disordering scenarios, where the unit cells are not placed perfectly and they are misaligned. This way, we investigate the electromagnetic properties of MMs when the arrays are defected. In this paper, we briefly report the accurate solutions of various real-life MM problems and present power transmission properties of these important structures.

\section{Modelling of Metamaterial Structures}

In this paper, SRRs and TWs are modelled by perfectly conducting sheets. Dimensions of the unit cells are in the order of microns to obtain negative effective permeability around $100 \mathrm{GHz}$ [3]. The SRR array depicted in Fig. 1(a) is constructed by the arrangement of $11 \times 18$ SRRs. Due to the negative effective permittivity stimulated in the medium, the transmission through the array is expected to decrease significantly around the resonance frequency. As depicted in Fig. 1(b) we construct multilayer SRR arrays as well as multilayer CMM structures using TWs in addition to SRRs. Dimensions of the TWs are compatible with the dimensions of the SRRs so that the CMM structures in Fig. 1(b) are expected to show double negativity around $100 \mathrm{GHz}$. In addition to multilayer structures, we also consider various disordering scenarios as depicted in Fig. 2. We specifically investigate the variation in the electromagnetic properties of the SRR arrays when the unit cells are not placed perfectly as in Fig. 2(a), but they are misaligned as in Figs. 2(b)-(d). By employing EFIE, we accurately model these defects in the MM structures while the resulting dense matrix equations are solved easily by MLFMA.

\section{EFIE Solutions of Metamaterial Structures}

By the discretization of EFIE for the solution of MM structures, we obtain $N \times N$ dense matrix equations in the form of

$$
\sum_{n=1}^{N} Z_{m n}^{E} a_{n}=v_{m}^{E}, \quad m=1, \ldots, N
$$

where

$$
Z_{m n}^{E}=\int_{S_{m}} d \boldsymbol{r} \boldsymbol{t}_{m}(\boldsymbol{r}) \cdot \int_{S_{n}} d \boldsymbol{r}^{\prime}\left(\overline{\boldsymbol{I}}-\frac{\nabla \nabla^{\prime}}{k^{2}}\right) g\left(\boldsymbol{r}, \boldsymbol{r}^{\prime}\right) \cdot \boldsymbol{b}_{n}\left(\boldsymbol{r}^{\prime}\right)
$$

${ }^{\dagger}$ This work was supported by the Scientific and Technical Research Council of Turkey (TUBITAK) under Research Grant 105E172, by the Turkish Academy of Sciences in the framework of the Young Scientist Award Program (LG/TUBA-GEBIP/2002-1-12), and by contracts from ASELSAN and SSM. 


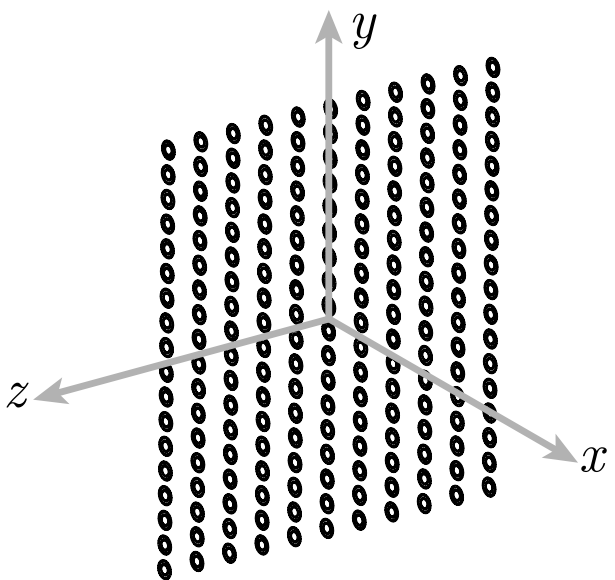

(a)

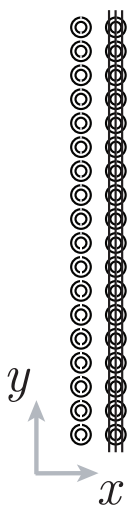

$x$

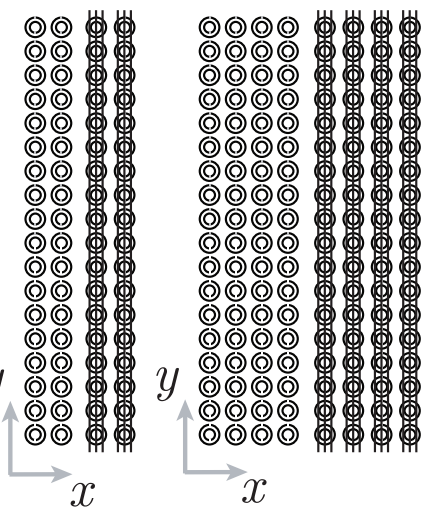

(b)

Figure 1: (a) Single-layer SRR array obtained by the arrangement of $11 \times 18$ SRRs. (b) 1-layer, 2-layer, and 4-layer SRR and CMM structures.

represents the matrix element, i.e., interaction of $m$ th testing and $n$th basis functions. We apply a Galerkin scheme and choose the basis functions $\boldsymbol{b}_{n}$ and testing functions $\boldsymbol{t}_{m}$ as Rao-Wilton-Glisson (RWG) functions [4]. In (2), $k$ is the wavenumber associated with the host medium and

$$
g\left(\boldsymbol{r}, \boldsymbol{r}^{\prime}\right)=\frac{e^{i k R}}{4 \pi R} \quad\left(R=\left|\boldsymbol{r}-\boldsymbol{r}^{\prime}\right|\right)
$$

denotes the homogeneous Green's function in frequency domain using phasor notation with the $e^{-i w t}$ convention. The $m$ th element of the excitation vector in (1) can be written as

$$
v_{m}^{E}=\frac{i}{k \eta} \int_{S_{m}} d \boldsymbol{r} \boldsymbol{t}_{m}(\boldsymbol{r}) \cdot \boldsymbol{E}^{\boldsymbol{i}}(\boldsymbol{r})
$$

where $\eta$ is wave impedance and $\boldsymbol{E}^{i}$ denotes the incident electric field. As it is commonly used in experimental setups [3], we choose the relative permittivity of the host medium as 4.8 .

Finding the coefficients $a_{n}$ in (1), we obtain the near-zone electric and magnetic fields as

$$
\boldsymbol{E}^{t}(\boldsymbol{r})=\boldsymbol{E}^{i}(\boldsymbol{r})+\sum_{n=1}^{N} \int_{S_{n}} d \boldsymbol{r}^{\prime}\left(\overline{\boldsymbol{I}}-\frac{\nabla \nabla^{\prime}}{k^{2}}\right) g\left(\boldsymbol{r}, \boldsymbol{r}^{\prime}\right) \cdot \boldsymbol{b}_{n}\left(\boldsymbol{r}^{\prime}\right) a_{n}
$$

and

$$
\boldsymbol{H}^{t}(\boldsymbol{r})=\boldsymbol{H}^{i}(\boldsymbol{r})+\sum_{n=1}^{N} \int_{S_{n}} d \boldsymbol{r}^{\prime} \boldsymbol{J}\left(\boldsymbol{r}^{\prime}\right) \times \nabla^{\prime} g\left(\boldsymbol{r}, \boldsymbol{r}^{\prime}\right) a_{n},
$$

respectively, where $\boldsymbol{H}^{i}$ is the incident magnetic field. Then, we define the power transmission at an observation point $\boldsymbol{r}$ as

$$
\mathcal{T}(\boldsymbol{r})=\frac{\mathcal{R} e\left\{\boldsymbol{E}^{t}(\boldsymbol{r}) \times\left[\boldsymbol{H}^{t}(\boldsymbol{r})\right]^{*}\right\}}{\mathcal{R} e\left\{\boldsymbol{E}^{i}(\boldsymbol{r}) \times\left[\boldsymbol{H}^{i}(\boldsymbol{r})\right]^{*}\right\}},
$$

where '*' represents the complex-conjugate operation. 


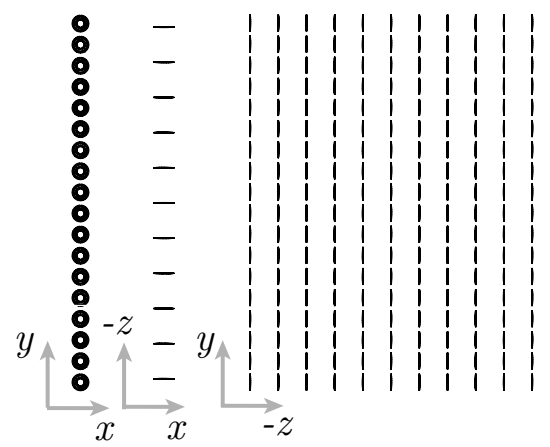

(a)

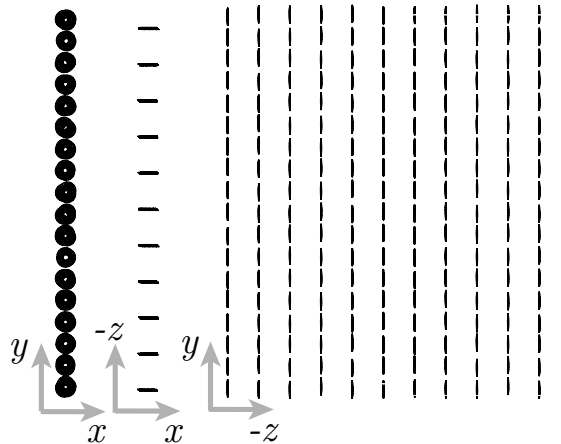

(c)

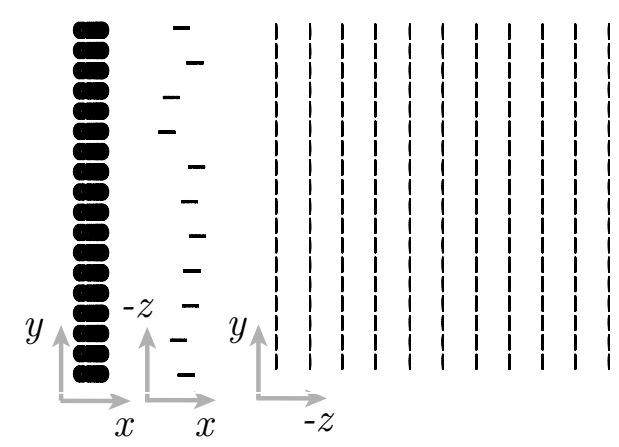

(b)

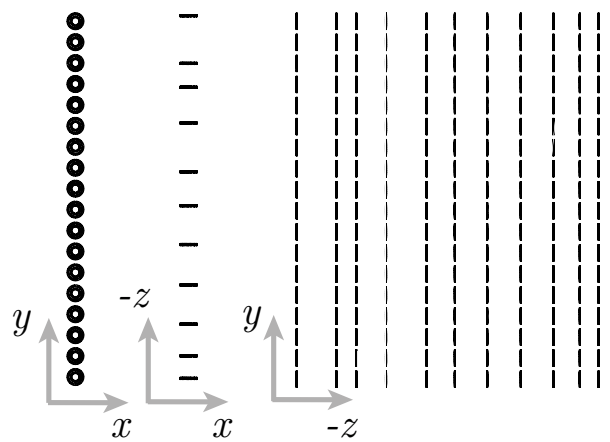

(d)

Figure 2: Various disordering scenarios for the $11 \times 18$ SRR array in Fig. 1(a). (a) Perfectly ordered array, (b) misalignments in the $x$ direction (X-disorder), (c) misalignments in the $x-y$ plane (Intraplane-Disorder: IP-disorder), and (d) misalignments in the $z$ direction (Z-disorder).

\section{Iterative Solutions by MLFMA}

Computation of each element of the dense matrix-equation in (1) requires the evaluation of a double integral over the testing and basis triangles. In order to solve large MM structures consisting of hundreds of unit cells, we employ iterative algorithms and accelerate the matrixvector multiplications via MLFMA, i.e.,

$$
\overline{\boldsymbol{Z}} \cdot \boldsymbol{x}=\overline{\boldsymbol{Z}}^{N F} \cdot \boldsymbol{x}+\overline{\boldsymbol{Z}}^{F F} \cdot \boldsymbol{x} .
$$

In MLFMA, only the near-field interactions denoted by $\overline{\boldsymbol{Z}}^{N F}$ are calculated directly and stored in the memory, while the far-field interactions are computed approximately in a group-by-group manner. Using a multilevel scheme, the overall complexity of the matrixvector multiplications is reduced to $O(N \log N)$, where $N$ is the number of unknowns.

For the iterative solutions of the problems involving MM structures, we consider preconditioners based on sparse approximate inverse (SAI) of the near-field matrix [5]. These robust preconditioners are required in order to obtain quick convergence for EFIE, which usually produces ill-conditioned matrix equations that are difficult to solve iteratively. On the other hand, increased resonance effects of the multilayer MMs lead to very ill-conditioned matrix equations, which need further information than that provided by the near-field matrix for a convergence. Then, we consider a two-level preconditioning technique based on an approximate MLFMA obtained by reducing the sampling rate of the original MLFMA for improved efficiency. 


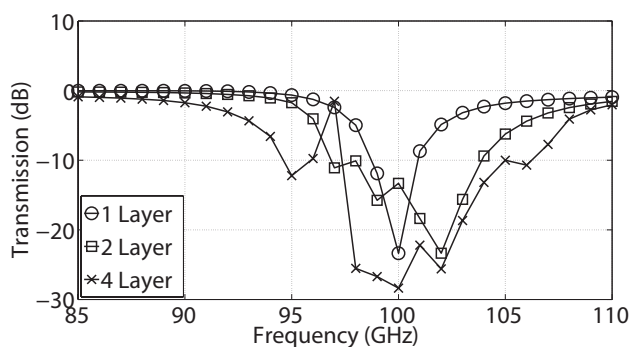

(a)

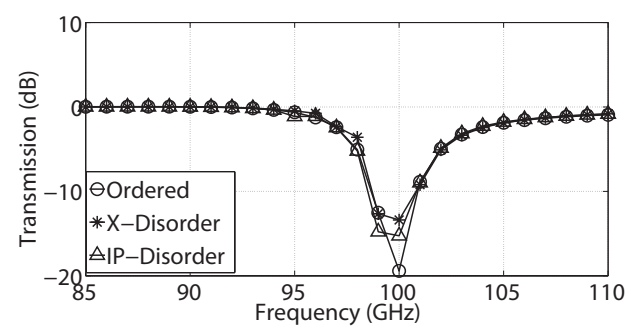

(c)

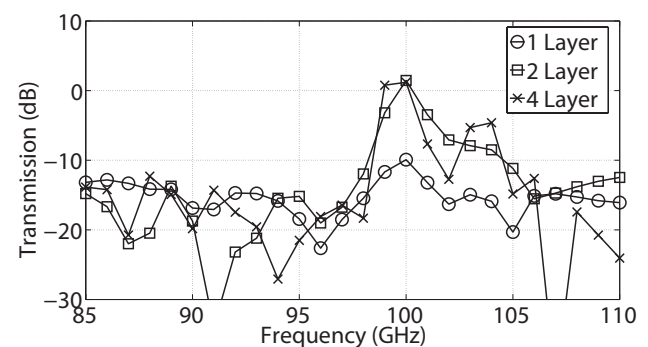

(b)

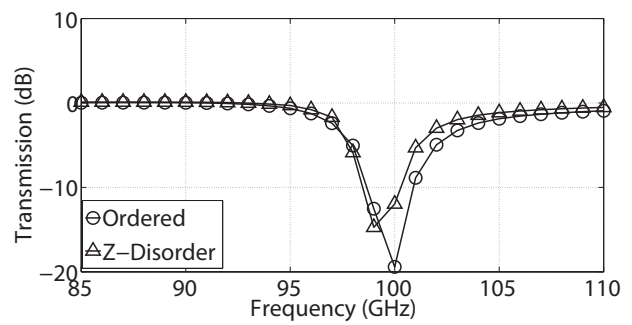

(d)

Figure 3: Power transmission for the SRR, CMM, and disordered SRR structures in Fig. 1(b) and Fig. 2.

\section{Results}

To investigate the transmission properties of the MMs in Fig. 1(b), we illuminate the structures by using a Hertzian dipole and solve the scattering problems using an MLFMA implementation. Figs. 3(a) and (b) depict the power transmission defined in (7) as a function of frequency for the SRR and CMM structures, respectively. We observe that SRR structures block the fields around $100 \mathrm{GHz}$, and this is mainly due to the negative effective permittivity introduced in the medium. On the other hand, the transmission through the CMM structures increase around $100 \mathrm{GHz}$ because of the double-negativity property. In other words, a CMM structure becomes transparent around $100 \mathrm{GHz}$, although its components, i.e., the SRR and TW arrays, block the fields at these frequencies. We also note that increasing the number of layers of the SRR structures extends the frequency range for the resonance effect. The double negativity of the CMM structures is also stronger when the structure is constructed by more than one layers as also depicted Fig. 3(b), where 1-layer CMM cannot provide 100\% (0 dB) transparency. Finally, Figs. 3(c) and (d) present the power transmissions for the various disordering scenarios in Fig. 2. Although the SRR array is significantly modified by various misalignments, we observe that the transmission properties are slightly affected. Among three different disordering schemes, the most effective one seems to be the Z-disorder, where the resonance frequency is shifted by a small amount as depicted in Fig. 3(d).

\section{References}

[1] A. J. Poggio and E. K. Miller, "Integral equation solutions of three-dimensional scattering problems," in Computer Techniques for Electromagnetics, R. Mittra, Ed. Oxford: Permagon Press, 1973, Chap. 4.

[2] W. C. Chew, J.-M. Jin, E. Michielssen, and J. Song, Fast and Efficient Algorithms in Computational Electromagnetics. Boston, MA: Artech House, 2001.

[3] M. Gokkavas, K. Güven, I. Bulu, K. Aydın, R. S. Penciu, M. Kafesaki, C. M. Soukoulis, and E. Özbay, "Experimental demonstration of a left-handed metamaterial operating at $100 \mathrm{GHz}$," Phys. Rev. B., vol. 73, no. 193103, 2006.

[4] S. M. Rao, D. R. Wilton, and A. W. Glisson, "Electromagnetic scattering by surfaces of arbitrary shape," IEEE Trans. Antennas Propagat., vol. AP-30, no. 3, 409-418, 1982.

[5] T. Malas and L. Gürel, "Sparse approximate inverse preconditioning in the context of multilevel fast multipole algorithm," research report, Bilkent University, Ankara, Turkey. 